\title{
Concepciones de ciencia, metacognición y autorregulación
}

\section{Rafael Lucio Gil}

Investigador del Instituto de Educación de la UCA (IDEUCA) “Xabier Gorostiaga, s.j.”

\section{Síntesis}

En este artículo se presentan los principales elementos teóricos sobre el enfoque de ciencia y sus implicaciones en la enseñanza. Su contenido responde a los resultados de un proceso de investigación realizado con varios profesores del área con la finalidad de provocar cambios relevantes en sus concepciones de ciencia y de su enseñanza. Sobresale en el estudio la aplicación de estrategias metacognitivas y autorreguladoras y los resultados que se obtienen al provocarse cambios relevantes en sus concepciones y prácticas.

\section{Introducción}

El propósito de este artículo es argumentar la importancia que tiene que la formación docente en ciencias incorpore el análisis de las concepciones que tienen los profesores sobre la ciencia para actuar sobre ellas con estrategias metacognitivas y autorreguladoras, de manera que logren superarlas adoptando un enfoque de ciencia desde la Nueva Filosofía de la Ciencia del Giro Cognitivo. Se presentan los resultados de una experiencia investigativa y formativa con profesores de ciencias a lo largo de un año con esta perspectiva, la que proporciona resultados de cambio de gran interés y significación. Esta experiencia parte de considerar que la concepción de ciencia se refleja en la visión que el profesorado tiene del conocimiento que enseña, la metodología de resolución de problemas y el modelo de interacción en el aula. Tales ejes logran ser dinamizados por el ejercicio de la actividad metacognitiva y la autorregulación y, sobre el plano que forman los tres, descansa la posibilidad de construir una nueva perspectiva epistemológica de la formación docente en ciencias. Los profesores poseen esquemas y concepciones sobre el conocimiento científico que enseñan, sobre su historia y desarrollo, su enfoque, los métodos que los acompañan, los actores que los construyen y la manera de enseñarlos en el aula. La concepción prevaleciente sobre el conocimiento y los procesos científicos interactúa, a su vez, con las ideas y prácticas que el profesorado manifiesta en la resolución de problemas. Como podrá verse, la perspectiva que tienen sobre la ciencia, sus métodos, su enseñanza y la formación del conocimiento, inducen al profesorado a asumir determinadas visiones, concepciones y rutinas prácticas en la enseñanza. Los métodos de resolución de problemas, que habitualmente han sido enseñados a los docentes y que éstos reproducen, aunque debieran inspirarse en el trabajo de los científicos, en la práctica son portadores de profundas contradicciones, mostrando abiertas diferencias y contraposiciones con el "espíritu científico" que debiera promoverse

1 BACHELAR, G. (1978). El espíritu científico. Buenos Aires: Siglo XXI. 
en el aula. Esta realidad, en buena parte, posiblemente aparezca estrechamente vinculada al grado de transposición didáctica que logran y el vínculo con la visión de la ciencia escolar. ${ }^{2}$ Partimos del supuesto que, en tanto el profesorado logra transformaciones significativas en su concepción sobre la ciencia y la enseñanza, en mayor medida logra reflexionar e intuir caminos nuevos en su enseñanza.

\section{Una marco referencial}

Una de las líneas de investigación más importantes en didáctica de las ciencias en los últimos años es el estudio del enfoque de ciencia, del conocimiento científico, de sus procesos y del papel de los científicos como inspiradores de la actuación didáctica del profesorado, y por ende, de su formación. La complejidad de esta perspectiva epistemológica también se expresa en la diversidad de enfoques, corrientes y modelos explicativos desarrollados por la Historia de la Ciencia, la Nueva Filosofía de la Ciencia y la Sociología de la Ciencia. No obstante esta complejidad, el debate internacional y sus implicancias en el terreno de la didáctica y de la formación del profesorado, han alcanzado consensos importantes que aportan a una nueva concepción y práctica de formación. El estudio de la Historia y la Filosofía de la Ciencia, en los últimos años, ha desarrollado un intenso debate teórico en la búsqueda de cimentar posturas de amplio consenso científico con mayor poder explicativo. Son múltiples los aportes científicos publicados en las últimas décadas desde diversas vertientes entre sí complementarias y a veces contradictorias ${ }^{3}$. Este debate, a su vez, ha proporcionado a la didáctica de las ciencias y a la formación del profesorado, nuevos referentes epistemológicos para ubicar y comprender las concepciones que posee el profesorado y sus influencias en la actuación didáctica en las aulas ${ }^{4}$.

El afán por sobreponerse a los mitos y creencias en todo tiempo, ha propiciado un acercamiento cada vez mayor al conocimiento científico, aunque nuevas creencias y mitos parecen haberlos reemplazado. La Historia de la Ciencia ha sido objeto de distintos modelos de análisis que van, con sus matices, desde el internalismo - para el que la ciencia se desarrolla por sus propias reglas internas- hasta el externalismo de origen marxista -para el que la ciencia está determinada por los acontecimientos de su tiempo conviertiéndola "en un mero epifenómeno de las condiciones económicas y sociales"5. Estos enfoques extremos encuentran hoy un punto de equilibrio con una visión más ecléctica de la historia de la ciencia, que considera la existencia de interacciones complejas entre ambos ámbitos. Esta propuesta de formación se inspira en este equilibrio presentando a los profesores una historia más contextualizada y poseedora de dinámicas específicas. Desde la perspectiva

2 IZQUIERDO, M. (1996). Relación entre la historia y la filosofía de la ciencia y la enseñanza de las ciencias. Alambique. Didáctica de las ciencias experimentales, $\mathrm{N}^{\circ} 8$, pp: 7-21.

3 Véase: BUNGE, M. (1973). La ciencia: su método y su filosofía. Buenos Aires: Siglo XX; BUNGE, M. (1978). Filosofía de la Física. Ciencia de la Ciencia. Barcelona: Ariel; ECHEVERRÍA, J. (1998). Filosofía de la ciencia. Madrid: Akal; GIERE, R.N. (1988). Toward a unified cognitive theory of science. Fotocopia, UAB; y GIERE, R.N. (1992). Cognitive Models of Sciences. Fotocopia, UAB.

4 ACEVEDO, D, A., SÁNCHEZ, H, A. (1990). Sobre el aprendizaje de los conceptos de la Mecánica. Revista Española de Física, 4(2), pp. 38-42; ASTOLFI, J.P., DAROT, E., GINBURGER-VOGEL, Y., TOUSSAINT, J. (1997). Practiques de formation en didactique des sciences. Belgique: De Boeck Université, Practiques Pedagogiques ; CAMPANARIO, J.M. (1998). Ventajas e inconvenientes de la Historia de la Ciencia como recurso en la Enseñanza de las Ciencias. Revista de Enseñanza de la Física, Vol. 11, No 1, pp. 5-14.

5 MIKULINSKI, S.R. (1989). La controversia internalismo externalismo como falso problema. En: Saldaña, J.J. Introducción a la teoría de la Historia de las ciencias. México: UNAM: p 232. 
Tradicional de la Ciencia, ampliamente extendida en el profesorado y con fuerte influencia en los enfoques didácticos, se considera que el conocimiento científico procede de la observación, la experimentación o la razón, según se trate de cualquiera de sus principales tendencias: inductivismo, empirismo y racionalismo lógico. El inductivismo parte de la objetividad de la observación y del razonamiento. La ciencia parte, por consiguiente, de enunciados observacionales, eludiendo la teoría que precede y determina a la observación. Para Chalmers esta perspectiva "está muy equivocada y es peligrosamente engañosa" ${ }^{\text {, }}$ estando su programa, desde la perspectiva lakatosiana ${ }^{7}$, en vías de degeneración, aún cuando sus aportes a la formalización de la ciencia han sido evidentes. El contraste entre el inductivismo y el constructivismo es evidente, en tanto para éste la ciencia empieza con teorías que preceden a los enunciados observacionales. "Conocemos sobre un conocimiento anterior", asegura Bachelard ${ }^{8}$, por lo que los enunciados que son resultado de las observaciones están influidos por la teoría. En el Novum Organum de F. Bacon para el empirismo, en el Discurso del Método de R. Descartes y La Crítica de la Razón Pura de E. Kant para el racionalismo, se encuentran los principales referentes clásicos de estas escuelas de pensamiento. Para los empiristas, la percepción es la fuente y prueba última del conocimiento, la experiencia es la única fuente del conocimiento, y las teorías se formulan a partir de la inferencia inductiva. Asume que la mente humana es como "una hoja en blanco" en la que se escribe la experiencia. Reichenbach ${ }^{9}$ refiere la burla que Bacon hacía de los racionalistas comparándolos con los empiristas. Los empiristas lógicos enfatizan el análisis lógico y lingüístico y reducen las ciencias a sistemas hipotéticos - deductivos, destacando la coherencia interna y la correspondencia entre las construcciones formales y los hechos en sí. El Racionalismo parte del poder absoluto de la razón para determinar el conocimiento verdadero. En su etapa de positivismo lógico o ultrainductivismo, iniciado por el Círculo de Viena en 1929, aplicó a la ciencia los métodos del análisis lógico. Esta perspectiva para Chalmers no es sino "una forma extrema de empirismo"10. El positivismo actual sigue presentando una imagen de la ciencia cargada de eficiencia a toda prueba ${ }^{11}$. La considera como un sistema autónomo aislado del mundo, un conocimiento intrínseco, puro y absoluto. Con esta lógica, la ciencia camina sobre bases seguras a partir de la observación y la experimentación como bases de un método científico del que siempre se derivan teorías fiables. Como sistema cerrado dispone de una lógica que impera, controla y decide "como un monarca absoluto y sin restricciones ${ }^{\prime 12}$. Esta visión sirve a los propósitos de una doble dominación $^{13}$ : una de tipo tecnológico, en cuanto el conocimiento científico se distribuye en forma desigual y no democrática; y otra de carácter tecnocrático, en tanto evidencia una fuerte jerarquización del saber científico. Esta perspectiva tradicional ha dado paso a visiones más amplias, con mayor poder explicativo, ajustadas a la realidad de la construcción del conocimiento científico.

\footnotetext{
CHALMERS, A. (1992). La ciencia y cómo se elabora. Madrid: Siglo XXI: p 24.

LAKATOS, I. (1987). Matemática, ciencia y epistemología. Madrid: Alianza Universidad.

BACHELARD, G. (1978). El espíritu científico. Buenos Aires: Siglo XXI.

REICHENBACH, H., (1985). La Filosofía científica. México: Fondo de Cultura Económica.

10 CHALMERS, A. (1992). La ciencia y cómo se elabora. Madrid: Siglo XXI: p7.

11 BERNAL, J. (1960). La ciencia en nuestro tiempo, México: UNAM.

12 MIKULINSKI, S.R. (1989). La controversia internalismo externalismo como falso problema. En: Saldaña, J.J. Introducción a la teoría de la Historia de las ciencias. México: UNAM: p 232.

13 GIORDAN, A. (1994). La enseñanza de las ciencias. Siglo XXI. En: I. M. J., Castelló, I. M. J.(Comp.). Dossier Maestría en Educación y Didácticas Especiales. Didáctica de las Ciencias Experimentales, Barcelona: UAB, Managua.
} 
El campo incipiente de la "ciencia de la ciencia" ha impactado, con sus análisis, radicalmente el panorama de la ciencia, lo que ha propiciado en los últimos cuarenta años una amplia gama de corrientes con diferentes matices y posiciones, en la mayoría de los casos complementarios. Estas corrientes forman parte del debate de lo que se ha dado en llamar Nueva Filosofía de la Ciencia. La ciencia, desde esta perspectiva, es estudiada en sentido holístico como un todo único ${ }^{14}$, aunque su actitud relativizadora no toma en cuenta la vertiente cognitiva. Sus principales aportes ayudan, también, a comprender los procesos de cambio de los profesores en este plan de formación. Desde este enfoque, la ciencia produce conocimientos válidos y útiles, producto del pensamiento humano, sujetos, por tanto, al cambio, al desarrollo permanente de nuevos razonamientos y modelos ${ }^{15}$, producto de la combinación del descubrimiento de hechos subjetivos sobre el mundo y la creación de nuevos conceptos. Para K. Popper ${ }^{16}$ es fundamental la actividad falsacionista que permite reemplazar una teoría por otra mejor con mayor capacidad explicativa y resistencia a las pruebas. Las concepciones postanalíticas alternativas están representadas por Kuhn, Toulmin, Lakatos, Feyerabend y otros. Todos coinciden en la ciencia y sus métodos como empresa compleja, inserta en la cultura y el contexto social, con carácter dinámico y en constante transformación. Para Lakatos ${ }^{17}$, una teoría no se considera falsada aún con evidencias en su contra, requiriendo más bien aumentar su poder explicativo, lo que es posible si se desarrolla otra teoría que compita con ella. Para la lógica kuhniana, el surgimiento de un nuevo paradigma afectará la estructura del paradigma vigente, atrayendo hacia sí a la mayoría de los científicos. La Ciencia de la Complejidad representa un paso audaz en el plano científico y metacientífico para la reinterpretación de la ciencia desde coordenadas diferentes a las del determinismo, el positivismo y el mecanicismo laplaciano ${ }^{18}$. Mientras el determinismo laplaciano presume, con los datos y condiciones iniciales requeridas, de conocer el pasado y el futuro, la Ciencia de la Complejidad advierte que no es posible predecir y controlar la evolución de los sistemas dinámicos reales, puesto que, en su mayoría, evolucionan de manera no lineal, desarrollando fases caóticas predecibles únicamente en términos probabilísticos o cualitativos. La Nueva Filosofía de la Ciencia del Giro Cognitivo presidida por Duschl y Giere e Izquierdo ${ }^{19}$, representa un paso más en el debate actual de la Filosofía de la Ciencia, con aportes importantes a la Didáctica de las Ciencias. Desde la teoría cognitiva aplicada a la construcción del conocimiento científico en la historia se explica de qué manera los científicos utilizan sus capacidades psíquicas para interactuar y construir la ciencia. La ciencia, como acto cognitivo, está sujeto a errores, por lo que los científicos son observadores que pueden ver el mundo desde sus propios puntos de vista ${ }^{20}$. Se ha pasado de una idea de ciencia como conjunto organizado y validado

14 MIKULINSKI, S.R. (1989). La controversia internalismo externalismo como falso problema. En: Saldaña, J.J. Introducción a la teoría de la Historia de las ciencias. México: UNAM: p 232.

15 BRUSH, S. (1991). Historia de la ciencia y enseñanza de las ciencias. Comunicación y Lenguaje, 11-12, pp: 174-177.

16 POPPER, K. (1967). La sociedad abierta y sus enemigos T.1 y 2. Buenos Aires: Paidós.

17 LAKATOS, I. (1987). Matemática, ciencia y epistemología. Madrid: Alianza Universidad.

18 PRIGOGINE, I., STENGERS, I. (1994). La nueva Alianza . Metamorfosis de la Ciencia. Madrid: Alianza Editorial.

19 DUSCHL, R, A. (1993). Research on the History and Philosophy of Science. En: Gabeld, A. (Ed.). Hanbook of Research on Science Teaching and Learning. New Yorrk: Macmillan Publishing Company; GIERE, R.N. (1988). Toward a unified cognitive theory of science. Fotocopia, UAB;

GIERE, R.N. (1992). Cognitive Models of Sciences. Fotocopia, UAB; GIERE, R.N. (1994). La Explicación Científica. México; IZQUIERDO, M. (1996). Relación entre la historia y la filosofía de la ciencia y la enseñanza de las ciencias. Alambique. Didáctica de las ciencias experimentales, $\mathrm{N}^{\circ} 8$, pp: 7-21.

20 KORTHALS, M. (1994). Reconstruction and explanation of conceptual development. Theory and Psychology, Sage, Vol, 4(1), pp. 6183. 
de conocimientos, a considerarla como un tipo de actividad humana, compleja y difícil de interpretar. Para Izquierdo, la propuesta actual entiende la "racionalidad fuerte" como una explicación inadecuada de la ciencia, asumiendo la "racionalidad moderada" como más contextual y pertinente para explicar la realidad científica. Los modelos cognitivos de ciencia dan sentido a los datos y muestran que la actividad científica es una actividad cognitiva al igual que lo es el aprendizaje. Giere $(1988,1992,1994)$ propone interpretar las teorías en sentido semántico más que formal, por cuanto han de permitir interpretar los fenómenos. Por ello, las teorías responden a "modelos teóricos” e "hipótesis teóricas" que las vinculan a los fenómenos. Giere (1999), uno de sus representantes más importantes, entiende que los "modelos teóricos" ocupan un lugar preferencial. Nuestro cerebro construye "representaciones mentales" o "mapas internos del mundo externo" sobre los fenómenos y los principios que los vinculan, uniéndolos mediante los conceptos.

En forma análoga, a los modelos imaginarios que todos construimos sobre las cosas, los modelos teóricos forman parte también de la imaginación, y sólo existen en la mente, en los escritos o en las descripciones verbales de los científicos. En esta nueva lógica, la ciencia encierra un significado distinto al convencional que siguiendo a Echeverría ${ }^{21}$ y a Izquierdo, puede resumirse en estas líneas de acción actuales: Las teorías son útiles a la acción tecnocientífica, los estudios de la ciencia tienen a una nueva filosofía práctica, los procesos de cambio científico son complejos, y la ciencia es "enseñable” y una actividad humana. La actitud metacientífica representa la "capacidad metacognitiva" que la ciencia desarrolla, y que la hace tomar conciencia de sí misma y de cómo lleva a cabo su cometido social. La inspiración que la filosofía de la ciencia ha identificado en la teoría cognitiva ${ }^{22}$, justifica el papel de la metacognición y la autorregulación como actuaciones estratégicas por antonomasia, capaces de aportar a una trayectoria exitosa a la ciencia escolar. De esta actitud metacientífica se nutre la ciencia escolar, al acercar la ciencia a la vida cotidiana del estudiantado, estableciendo pasarelas entre el saber sabio, el saber cotidiano y los mecanismos autorreflexivos y críticos que favorecen un encuentro exitoso entre ellos. El conocimiento científico a ser aprendido atiende a tres grandes dimensiones del saber: declarativa, procedimental y actitudinal, y posee un carácter eminentemente social compartido. Son múltiples los factores que interactúan en él: las concepciones epistemológicas dominantes en la comunidad científica y en quienes deciden el curriculum de ciencias, las relaciones culturales y sociales con la comunidad y las finalidades sociales e ideológicas asignadas a su enseñanza ${ }^{23}$.

\section{La formación docente requiere de una perspectiva epistemológica y didáctica} coherente con la forma como el alumno aprende ciencia: Entendemos la ciencia escolar como la ciencia cuyas características responden al medio escolar. No se trata, por tanto, de la ciencia de los científicos y es más que la suma de transposiciones didácticas de cada una de las ciencias, siendo el todo más que sus partes. Sus modelos teóricos no pueden ser los

21 GIERE, R.N. (1988). Toward a unified cognitive theory of science. Fotocopia, UAB;

22 GIERE, R.N. (1992). Cognitive Models of Sciences. Fotocopia, UAB; GIERE, R.N. (1994). La Explicación Científica. México

23 GIROUX, H.A. (1990). Los profesores como intelectuales. Hacia una pedagogía crítica del aprendizaje. Temas de Educación. Barcelona: Paidós, MEC; KEMMIS, S. (1988). El curriculum: Más allá de la Teoría de la Reproducción. Madrid: Morata; PORLÁN, A, R., RIVERO, A. (1998). El conocimiento de los profesores. Serie Fundamentos No 9. Colección Investigación y Enseñanza. Sevilla: Díada Editores; LEMKE, J.L. (1997). Aprender a hablar ciencia. Lenguaje, aprendizaje y valores. Temas de Educación, Barcelona: Paidós. 
mismos que los de una ciencia disciplinar, pues contribuye a afrontar la enseñanza desde una perspectiva de complejidad, con una forma de mirar determinada, un marco teórico propio, y una teoría de sistemas con sus preguntas, lenguajes, analogías, modelos y valores. Las finalidades del saber sabio y de la ciencia escolar son distintas, estando dirigida ésta a la enseñanza con una epistemología, unos valores y una práctica propios. Para Izquierdo, los modelos de ciencia escolar actualmente en uso y debate son diversos, por lo que mientras algunos modelos destacan más su dimensión práctica al margen de los referentes teóricos actuales, otros buscan un acuerdo entre los marcos teóricos de actualidad y sus componentes prácticos.

\section{Las creencias de los profesores sobre los procesos de la ciencia son obstáculos a} superar: Los principales aportes de los estudios sobre las creencias del profesorado sobre la ciencia, proporcionan referentes fundamentales para analizar las ideas de los profesores en el marco de esta propuesta de formación. Estas investigaciones tienen su origen en los estudios sobre las ideas de los alumnos. Al estudiarlas podremos también responder a este interrogante para los propósitos de esta propuesta: ¿Qué relaciones guardan estas concepciones con las prácticas de enseñanza? Los medios de comunicación y el lenguaje cotidiano son cruciales para moldear la conciencia tecnológica de la gente en general, originando tres mitos compartidos también por los profesores: el progreso científico es permanente y está asociado a la acumulación de cambios cuantitativos, las explicaciones científicas que divulgan los medios son razonables por sí mismas, y el mito de que la ciencia es infalible. Estos mitos ingresan también a los estilos de formación docente y a las aulas de clase, originando a su vez un espectro de concepciones derivadas que desvirtúan la visión de la ciencia, su enseñanza y los aprendizajes. Según Vázquez y Manassero ${ }^{24}$, la revisión realizada por Ommerod y Duckworth (1975) de 500 trabajos de investigación, muestra que la mayoría de textos revisados presenta deficiencias en el constructo ciencia y sus derivados. Las historias presentan una ciencia heroica, declarativa, políticas científicas correctas, y legitiman una filosofía de la ciencia tradicional25. Los trabajos revisados y la experiencia realizada al respecto coinciden en que los profesores ven la ciencia como neutral e imparcial ante las tensiones sociales, sin mantener vinculaciones ideológicas ni estar sometida a intereses; la ven exacta por utilizar un método preciso, atendiendo sólo cosas desconocidas, lógica, simplista y disponiendo de un método específico que la hace superior y diferente a las demás disciplinas ${ }^{26}$. Para Furió et $\mathrm{al}^{27}$. el autoritarismo en la educación científica es una característica del positivismo lógico hegemónico del siglo XX, ideología

24 VAZQUEZ, A, A., MANASSERO, M, M. A. (1995). Actitudes relacionadas con la ciencia: una revisión conceptual. Enseñanza de las Ciencias, 13, 3, pp. 337-346.

25 MILNE, C. (1998). Philosophically Correct Science Stories?. Examining the Implications of Heroc Science Stories for School Science. Journal of Research in Science Teaching, Vol. 35, No .3, pp. 175-187; PEDRINACI, E. (1994). Espistemología, historia de las ciencias y abejas. Investigación en la Escuela. No 23, pp. 95-102.

26 VAZQUEZ, A, A., MANASSERO, M, M. A. (1995). Actitudes relacionadas con la ciencia: una revisión conceptual. Enseñanza de las Ciencias, 13, 3, pp. 337-346; VÁZQUEZ, A, A., MANASSERO, M, M. A. (1999). Características del conocimiento científico: creencias de los estudiantes. Enseñanza de las Ciencias, 17(3), pp. 377-395; VAZQUEZ, A, A. (1994a). El paradigma de las concepciones alternativas y la formación de los profesores de ciencias. Enseñanza de las Ciencias, 12(1), pp. 3-14; VASQUEZ, A, A. (1994b). Concepciones iniciales sobre la enseñanza en profesores de ciencias de secundaria en formación. Revista Interuniversitaria de Formación del Profesorado, No 21, Sep/Dic., pp. 159-173; CAMPANARIO, J.M. (1999). La ciencia que no enseñamos. Enseñanza de las Ciencias, 17(3), pp: 397-410; MELLADO, J, V. (1996). Concepciones previas de los profesores en formación inicial de Primaria y Secundaria. Enseñanza de la Ciencias, 14(3), pp. 289-302.

27 FURIÓ, C., ET AL. (1992). La formación inicial del profesorado y las didácticas específicas. Investigación en la Escuela, № 16, pp. 9-21. 
cientifista resistente al criticismo y a aceptar el nuevo enfoque de la filosofía de la ciencia. Como consecuencia, el profesorado suele expresar una imagen positivista de ciencia sobre la base de su visión inductiva y superficial del método científico y la concepción objetivista del proceso científico.

La metacognición juega un papel central en la toma de conciencia de los procesos de pensamiento y acción del profesorado: Se orienta a desencadenar cambios autorregulados dirigidos a mejorar la actividad didáctica en el aula. En este empeño partimos del supuesto que las concepciones del profesorado sobre la ciencia, sus procesos de construcción y su enseñanza, determinan o condicionan los estilos de pensar y practicar la enseñanza, si bien, tal relación tiene un carácter complejo y no lineal. Hemos mostrado cómo los enfoques sobre la ciencia han evolucionado significativamente en la historia, lo que pone de manifiesto que la actividad metacientífica realizada por la filosofía, la historia y la sociología de la ciencia y la propia didáctica de la ciencia, ha tenido frutos importantes. Esta actitud metacognitiva de la propia ciencia ha provocado este proceso transformador al reflexionar sobre sus propios procesos, las relaciones entre los fenómenos, sus hipótesis y teorías, y los estilos de trabajo desarrollados por los científicos. Estas representaciones son comparables con "teorías" del saber común ${ }^{28}$ de las ciencias "populares" que se difunden en la sociedad, y que están compuestas por un "núcleo estable" y "elementos periféricos", lo que hace más probable su ubicación marginal y no tanto su cambio radical.

\section{Resultados}

\section{Evolución que sufren las concepciones de ciencias de los profesores participantes en la experiencia investigativa.}

De los casos estudiados, la mayoría, salvando sus diferencias, alcanza una evolución que los ubica con características del Perfil Progresivo del "Profesor Reflexivo Sistémico Investigativo" y "Reflexivo Sistémico". La clave fundamental de este desarrollo reside en el propio plan de formación en primera instancia: El nivel de interés que provocó el plan de la investigación, les motivó de forma especial: El enfoque de la ciencia y de resolución de problemas, temas para ellos más complejos y con mayores dificultades en el aula, $l a$ participación siempre asidua a los diálogos con el investigador y su compromiso para llevar a cabo los compromisos de estudio, el diario de campo que elaboraron los profesores en el desarrollo de su capacidad reflexiva y comunicativa de los cambios, la realización de la diagnosis de las ideas alternativas de sus alumnos y de la unidad didáctica requirió de ellos mayor nivel de compromiso y dedicación, el ejercicio de la reflexión tuvo intensidad y continuidad logrando un perfil progresivamente investigativo, e hicieron mayor uso del lenguaje escrito y verbal con reflexiones sobre sus experiencias. Otras razones dependientes de los contextos de los profesores también influyeron: Las características de su formación antecedente, sus contextos profesionales e institucionales, los tiempos dedicados, las características de personalidad y de enfoques filosóficos de su profesión.

28 MOSCOVICI, S. (1998). Comment voit-on le monde?. Représentations sociales et réalité. 


\section{Del positivismo e inductivismo a una visión social y constructiva}

Llama la atención cómo los profesores participantes que inician el proceso investigativo y formativo en el nivel más bajo de "Profesor Reflexivo Externalista Acrítico", llegan a alcanzar los máximos niveles en su evolución, especialmente por la incorporación que hacen del nuevo enfoque de ciencia. Todos los profesores ingresan al plan con una idea de ciencia que posee rasgos positivistas e inductivistas, más o menos explícitos y coherentes, diferenciándose en matices; en todos ellos, las anécdotas de historia de la física ocupan un lugar para motivar al aprendizaje. Este enfoque de la ciencia evolucionó al máximo nivel de desarrollo ubicándose en el Perfil Progresivo de "Profesor Reflexivo Sistémico Investigativo". El hecho de que los casos hayan superado su idea inicial de ciencia y logrado incorporar en distintos grados el enfoque en el aula, es una muestra que la estrategia de la investigación con su plan de formación obtiene excelentes resultados. Además, queda demostrado cómo a través del estudio, la reflexión y el debate sobre los rasgos del nuevo enfoque, los profesores acaban incorporando gran parte de estos rasgos, invadiendo con él de manera explícita en unos casos e implícita en otros, la enseñanza, la interacción y la resolución de problemas en el aula.

\section{De la transmisión del conocimiento a una enseñanza centrada en la superación de las ideas alternativas de los alumnos}

Es interesante resaltar que los profesores logran una relación explícita entre su enfoque de ciencia y la práctica de su enseñanza, pudiéndose apreciar que todos logran progresar en su idea de ciencia. Sin embargo, aunque el profesorado hable de su nueva idea de ciencia, no necesariamente este cambio involucra su práctica didáctica. Cambiar la enseñanza de ciencias es, para todos los casos estudiados, una preocupación casi obsesiva, considerando los malos resultados que obtienen con sus alumnos, siendo coincidentes, al ingresar al plan, en su visión transmisora - receptora con ligeras variantes. En todos los casos, el enfoque inicial de ciencia y del conocimiento guarda relación con la idea que poseen sobre la enseñanza, evolucionando hacia un enfoque constructivo, procedente de dos afluentes: el nuevo enfoque de ciencia, y la fuerza que aporta a su visión cognitiva y epistemológica la indagación que hacen de las ideas de los alumnos. En todos los casos, la idea sobre la enseñanza se transforma desde su visión inicial a una visión más centrada en los alumnos, teniendo en cada caso sus especificidades, lo que les compromete a indagar sus ideas alternativas y, a partir de sus lógicas, elaborar actividades de aprendizaje. Puede decirse que, en la medida que adquieren mayor conciencia sobre las ideas de sus alumnos, van conformando una representación más clara del carácter constructivo del aprendizaje e identificando, como mediación, un nuevo estilo de interacción más centrado en los estudiantes.

El proceso investigativo y de formación pone de manifiesto que existe un obstáculo epistemológico que afecta a los profesores participantes y que parece funcionar en tres eslabones articulados entre sí: conectar con éxito las nuevas concepciones con los medios didácticos en la planificación, interconectar estos medios con su aplicación práctica, y conectar la percepción de insuficiencias con las adecuaciones a la Zona de Desarrollo Próximo de sus alumnos. 
En todos los casos se perciben algunos facilitadores de los cambios que contribuyen a que superen los obstáculos: Su interés por cambiar y promover una relación horizontal en el aula, aparece vinculado al interés que despiertan en ellos las ideas alternativas de sus alumnos y la práctica de la reflexión. Resalta la facilidad de comprensión y asunción de aspectos del nuevo enfoque de ciencia. Los diálogos posibilitaron la apertura de los profesores a compartir sus experiencias, lográndose un

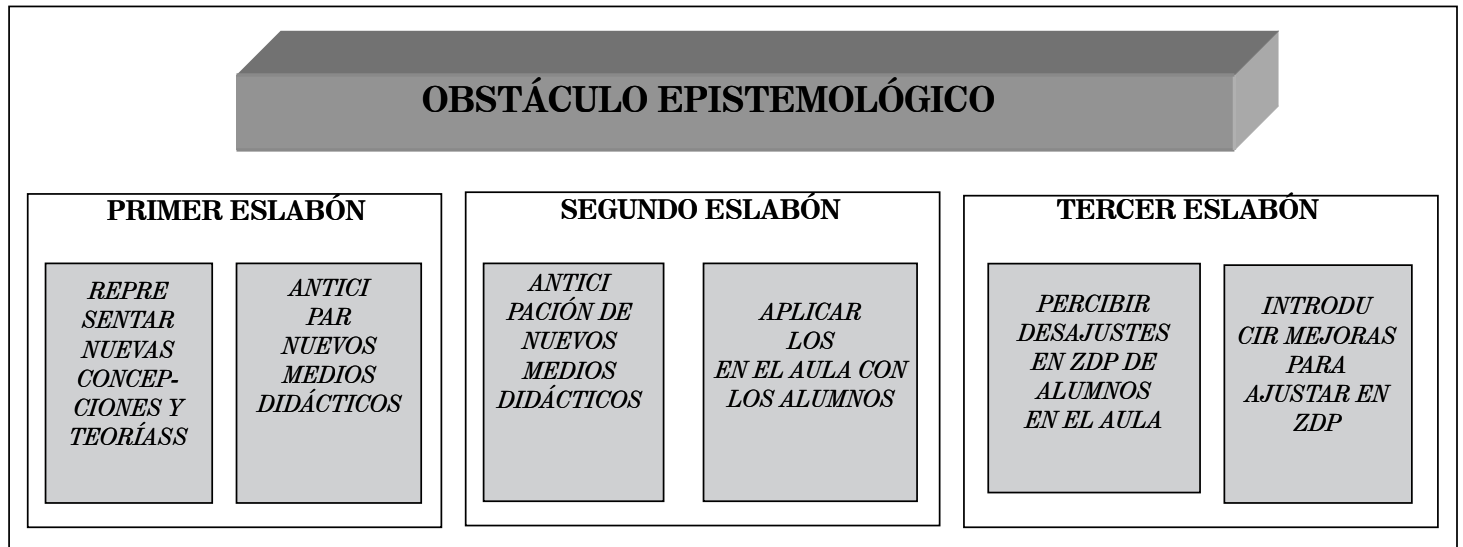

mayor nivel de transparencia sobre éstas, llevando su reflexión a criticar sus actuaciones de manera directa. Las actividades didáctica que realizaron influyeron de manera determinante para que canalizaran su interés hacia las ideas de los alumnos, lo que provocó en ellos cambios significativos en sus formas de entender y practicar la enseñanza y el enfoque de ciencia. El proceso interactivo y metarreflexivo, a la vez que se produce, reviste singular importancia por el impacto que tiene en sus cambios.

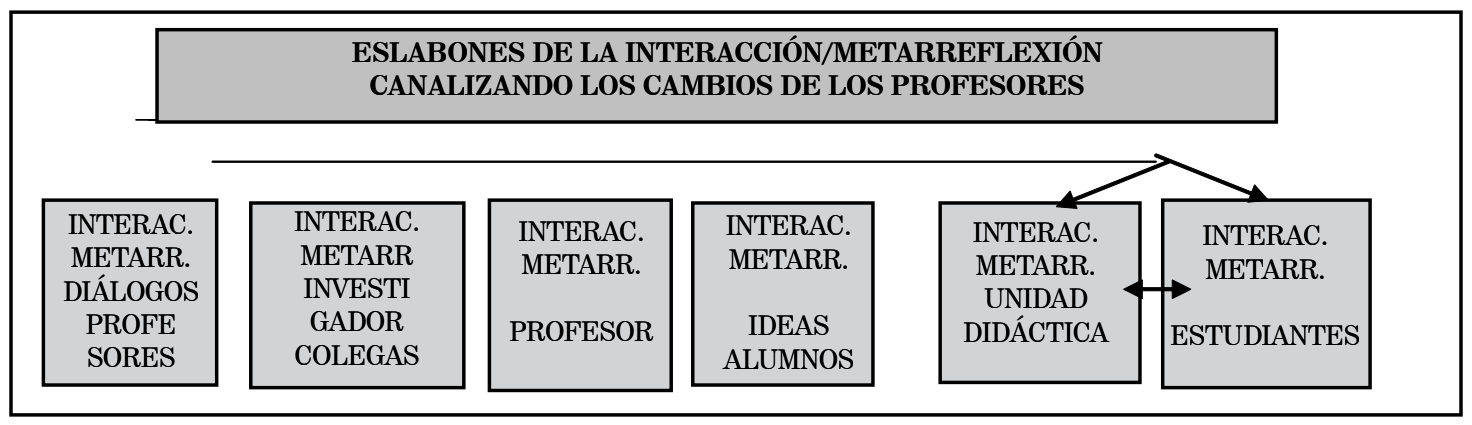


Los tres eslabones se fruncen así: la interacción que tienen con el investigador y los colegas; la interacción entre los profesores y las ideas alternativas de los alumnos; y la interacción entre el diseño didáctico innovador y los estudiantes en el aula.

\section{A título de Conclusión}

A partir de estos resultados es posible palpar el efecto positivo que tiene en los cambios de los profesores la práctica sistemática de la actividad metacognitiva, principalmente en su concepción de ciencia. También se pone de manifiesto que todos los participantes logran avanzar significativamente en sus niveles de reflexión metacognitiva y autorregulación de las prácticas, en tanto experimentan avances en sus procesos de modelización de la reflexión, tanto en intensidad como en niveles de calidad. A pesar de ello, se ponen de manifiesto un conjunto de factores que obstaculizan los cambios, y que tienen un carácter epistemológico, y otros son de tipo antropológico-histórico y también didáctico. Así mismo, se desvelizan un conjunto de facilitadores que contribuyen a que los cambios se den con mayor prestancia, como es la actitud del formador en cuanto a su transparencia y horizontalidad, y sus avances en la concepción de ciencia.

Los profesores tienen ideas alternativas sobre la ciencia que enseñan. Éstas son de diverso tipo y se asocian a diversos enfoques que ha tenido la epistemología de la ciencia. Estas ideas determinan o condicionan el enfoque de su enseñanza. Experiencias e investigaciones desarrolladas demuestran en diversos contextos la influencia que estas ideas alternativas tienen en la manera como los profesores conciben el conocimiento científico que enseñan, e incluso, la actitud y métodos didácticos que utilizan en el aula. El enfoque de la Filosofía de la Ciencia del Giro Cognitivo reúne el carácter relativizador, la interacción de los contextos y el perfil cognitivo del científico. Es el enfoque más cercano a la autenticidad de la ciencia. Su perfil de "racionalidad moderada" demuestra reconocimiento, por una parte, de la influencia que la sociedad y el entorno tienen en el desarrollo de la ciencia, pero también la capacidad que ésta tiene para aportar a este desarrollo, a la vez que también toma en consideración las características cognitivas que posee el científico. En este sentido, se aproxima con mayor realismo a una actividad didáctica que se ubica en un contexto definido, pero en el que confluyen influencias no sólo externas, sino también internas al profesor y sus estilos cognitivos de pensamiento. La Metacognición y Autorregulación, se constituyen como estrategias privilegiadas para provocar cambios relevantes en las visiones de ciencia de los profesores. Al activar las estrategias, los profesores logran comparar sus concepciones con las maneras como la ciencia desarrolla su quehacer y con sus producciones, de manera que se motivan a mejorar sus representaciones de los procesos científicos, y en consecuencia, a mejorar las estrategias de enseñanza. El enfoque de la formación ha de tomar en cuenta las preconcepciones docentes y enfocarse en una visión más innovadora de la ciencia. Es importante reconocer que los planes de formación inicial y permanente de los profesores de ciencias, no tienen incorporada de forma expresa o como eje transversal esta perspectiva tan importante. 


\section{De estos resultados se desprenden recomendaciones}

Es preciso transformar las concepciones que suelen presidir la formación y capacitación habituales, las que se caracterizan por su perspectiva instrumental; se trata de adoptar una nueva perspectiva de la formación, dirigida por una concepción y práctica que parta de las concepciones de los profesores, activando sus capacidades metacognitivas y autorreguladoras, en orden a reformular sus concepciones y prácticas. La investigación muestra, por otra parte, que dado que los obstáculos epistemológicos son evidentes, es necesario que la formación y capacitación se repiensen en términos que sirvan para que los profesores logren sobrepasar tales obstáculos en todos sus eslabones, lo que exige organizar la formación para superar estos obstáculos. De igual forma, al existir facilitadores de la formación, es preciso que éstos se logren incorporar en los procesos de formación. En síntesis, la formación y capacitación docentes deberán estar dominadas por la aplicación sistemática de estrategias metacognitivas y autorreguladoras y centrar su interés en que los profesores transformen sus concepciones de ciencia para así mejorar su enseñanza. 